\title{
TRISEGMENTECTOMIA HEPÁTICA DIREITA POR VIDEOLAPAROSCOPIA
}

\section{LAPAROSCOPIC RIGHT TRISECTIONECTOMY}

\author{
Marcel Autran C. Machado, TCBC-SP'; Fábio F. Makdissi²; \\ Rodrigo C.T. Surjan ${ }^{3}$; André C. Oliveira²; Victor F. Pilla, ACBC-SP'; \\ Antônio R. Teixeira ${ }^{2}$,TCBC-SP²
}

\begin{abstract}
RESUMO: Introdução: Em 2007 os autores descreveram a primeira hepatectomia direita por videolaparoscopia realizada no Brasil. Hepatectomia direita ampliada, também conhecida como trisegmentectomia direita, é procedimento altamente complexo e implica em grande retirada do volume hepático. Os autores descrevem a primeira trisegmentectomia direita por videolaparoscopia realizada no Brasil. Técnica: O paciente é colocado em posição supina em decúbito lateral esquerdo. O cirurgião se coloca entre as pernas da paciente. Utilizamos cinco trocartes, três de $12 \mathrm{~mm}$ e dois de $5 \mathrm{~mm}$. Devido à embolização prévia da veia porta direita, o hilo hepático não é dissecado. O pedículo portal direito é seccionado com grampeador laparoscópico de carga vascular por meio de acesso intra-hepático, segundo técnica previamente descrita pelos autores. A seguir procede-se a mobilização do fígado direito seguido de dissecção da veia cava retro-hepática e secção da veia hepática direita. Estes passos são realizados sem manobra de Pringle. O fígado é seccionado com combinação de bisturi harmônico e grampeador endoscópico. O pedículo do segmento 4 é seccionado dentro do fígado. O espécime é retirado por meio de incisão supra-púbica e a área cruenta é revista para verificar hemostasia. O procedimento é encerrado e dreno de sistema fechado é posicionado junto à área cruenta. Conclusão: Trisegmentectomia hepática direita por videolaparoscopia é procedimento factível e seguro e deve ser considerado para pacientes selecionados. Este procedimento deve ser realizado em centros especializados e por cirurgiões com experiência tanto em cirurgia hepática como cirurgia laparoscópica avançada (Rev. Col. Bras. Cir. 2008; 35(5): 338-341).
\end{abstract}

Descritores: Fígado; Laparoscopia; Trisegmentectomia; Anatomia; Técnica.

\section{INTRODUÇÃO}

Ressecção hepática é o tratamento definitivo de diversas doenças benignas e malignas do fígado. A maior experiência com a videocirurgia e recentes avanços técnicos de instrumental e técnica, levaram a um aumento no número de ressecções hepáticas por videolaparoscopia no Brasil e em diversos centros do mundo ${ }^{1-5}$. Entretanto, ressecções hepáticas laparoscópicas são procedimentos complexos e necessitam de conhecimento amplo em cirurgia hepática e laparoscopia avançada. Em 2007 os autores descreveram a primeira hepatectomia direita por videolaparoscopia realizada no Brasil ${ }^{1}$. Desde então houve um crescimento das indicações e no número de procedimentos hepáticos realizados por esta via.

Hepatectomia direita ampliada, também denominada por alguns autores, de trisegmentectomia direita, é um procedimento altamente complexo e implica em grande sacrifício do volume hepático ${ }^{6}$. Por este motivo só é realizado em centros especializados. A realização deste procedimento por via laparoscópica é ainda mais desafiadora. Do nosso conhecimento, existe apenas um relato da realização deste procedimento no mundo na literatura de língua inglesa ${ }^{7}$.

Os autores descrevem a primeira trisegmentectomia direita realizada totalmente por videolaparoscopia realizada no Brasil.

\section{TÉCNICA}

Devido ao grande volume hepático a ser removido por este método, é fundamental a realização de exames de imagem como tomografia ou ressonância com estudo da volumetria hepática total e volumetria do futuro remanescente hepático. Se o volume remanescente for menor a $30 \%$ do volume total do fígado, deve-se realizar embolização pré-operatória do ramo direito da veia porta com o intuito de causar uma hipertrofia compensatória do fígado esquerdo. Após cerca de quatro a seis semanas, repete-se exames de imagem e volumetria para confirmar o crescimento do fígado esquerdo.

O paciente sob anestesia geral é colocado em posição supina em decúbito lateral esquerdo. O cirurgião se coloca entre as pernas da paciente. O monitor é colocado atrás da cabeça do paciente.

O pneumoperitônio com $\mathrm{CO}_{2}$ é estabelecido com pressão abdominal de $12 \mathrm{mmHg}$. Utilizamos cinco trocartes, três de $12 \mathrm{~mm}$ e dois de $5 \mathrm{~mm}$ (Figura 1A). Os trocartes de $12 \mathrm{~mm}$ são necessários para o uso do grampeador endoscópico com carga linear. A cavidade abdominal e o fígado são visualizados com ótica de $30^{\circ}$.

Os ligamentos redondo e falciforme são seccionados rente à parede abdominal com o objetivo de facilitar ulterior fixação do fígado remanescente (Figura 1B). Os ligamentos falciforme e coronário são seccionados até exposição da veia

1. Professor Livre-Docente da Faculdade de Medicina da Universidade de São Paulo - USP.

2. Médico Assistente da Faculdade de Medicina da Universidade de São Paulo - USP

3. Médico Preceptor da Faculdade de Medicina da Universidade de São Paulo - USP.

Recebido em 16/05/2008

Aceito para publicação em 27/06/2008

Trabalho realizado no Departamento de Gastroenterologia do Hospital das Clínicas da Faculdade de Medicina da Universidade de São Paulo. Ver vídeo no Scielo. 
cava supra-hepática (Figura 1C). O fígado direito é mobilizado após secção do ligamento triangular direito (Figura 1D). O pedículo portal direito é seccionado com grampeador laparoscópico de carga vascular por meio de acesso intrahepático (Figura 2), segundo técnica previamente descrita pelos autores ${ }^{5}$. A seguir procede-se a mobilização do fígado direito seguido de dissecção da veia cava retro-hepática e secção da veia hepática direita (Figuras 3A e 3B). A face anterior da veia cava retro-hepática é amplamente exposta após secção das veias hepáticas acessórias oriundas dos segmentos seis e sete (Figura 3C). O tronco comum, incluindo as veias hepáticas média e esquerda, é circundado para

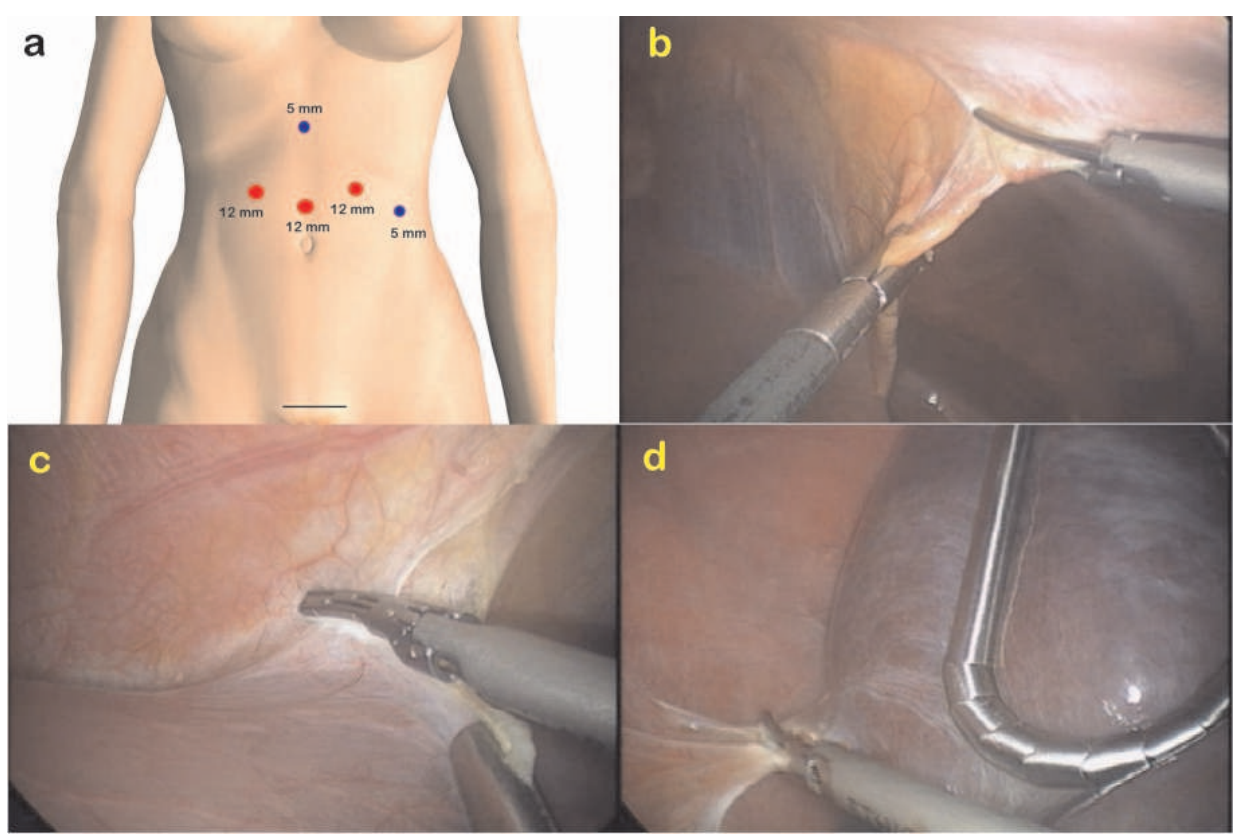

Figura 1 - Trisegmentectomia hepática direita por laparoscopia. a. Posicionamento e tipo de trocartes utilizados. A peça é retirada por meio de incisão supra-púbica feita ulteriormente. b. Os ligamentos redondo e falciforme são seccionados junto à parede abdominal para facilitar posterior fixação do fígado. c. Ligamentos falciforme $e$ coronário são seccionados até exposição da veia cava supra-hepática. $d$. Ligamento triangular direito é seccionado seguido de ampla mobilização do fígado direito. controle do retorno venoso hepático (Figura 3D). Esta manobra reduz o sangramento durante a secção do parênquima hepático. Estes passos são realizados sem manobra de Pringle. O fígado é seccionado com combinação de bisturi harmônico (LCS; Ethicon Endo Surgery Industries, Cincinnati, OH, USA) e grampeador linear endoscópico com carga vascular (Figura 4A e 4B). O pedículo do segmento quatro é seccionado dentro do fígado, durante a secção do fígado. O ligamento falciforme é suturado à parede abdomi- nal para manter o fígado remanescente na sua posição anatômica (Figura 4C) e evitar rotação do fígado esquerdo para o hipocôndrio direito que, se ocorrer, pode levar à dobra da veia hepática esquerda, levando agudamente à síndrome de Budd-Chiari ${ }^{8}$. O espécime (Figura 4D) é retirado por meio de incisão supra-púbica e a área cruenta é revista para verificar hemostasia.

O procedimento é encerrado e dreno de sistema fechado é posicionado junto à área cruenta.
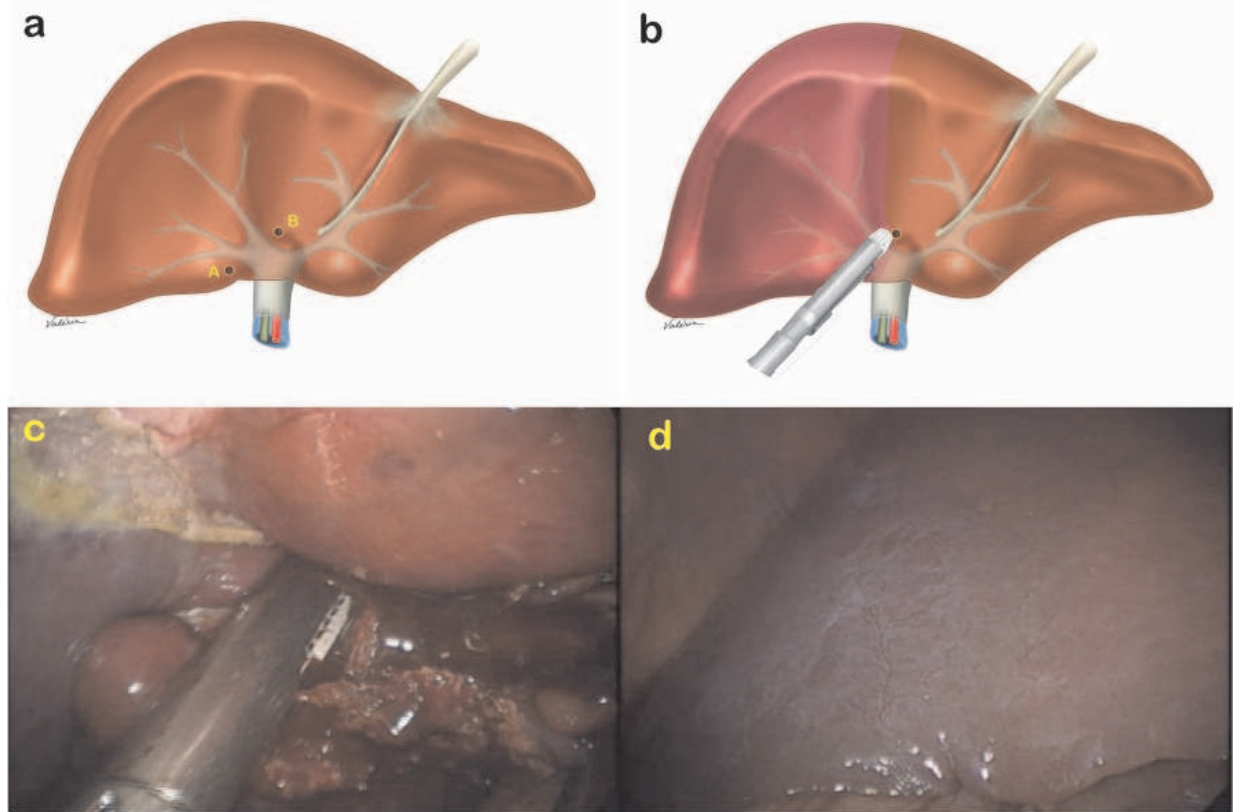

Figura 2 - Acesso intra-hepático ao pedículo Glissoniano direito. a. Desenho esquemático das incisões necessárias para acesso ao pedículo portal direito. b. Grampeador endoscópico linear com carga é introduzido através das incisões e uma vez fechado resulta em isquemia do fígado direito. c. Foto intra-operatória. Grampeador é disparado. Note fígado direito isquêmico e fígado esquerdo de coloração normal. d. Aspecto do fígado após secção do pedículo direito. 


\section{DISCUSSÃO}

O desenvolvimento da técnica de ressecção hepática por videolaparoscopia necessita de capacitação técnica em cirurgia laparoscópica avançada e em cirurgia hepática. A falta destes conhecimentos pode resultar em sangramento intraoperatório com risco de complicações graves e mortalidade.
A trisegmentectomia hepática direita envolve a ressecção dos segmentos 4, 5, 6, 7 e 8 e é considerada uma ressecção hepática maior ${ }^{6}$. A realização desta técnica por videolaparoscopia é um procedimento altamente complexo. Necessita de instrumentos especiais como o uso de grampeadores endoscópicos com carga vascular e do bisturi harmônico ${ }^{1,7}$. No entanto, os autores mostraram que

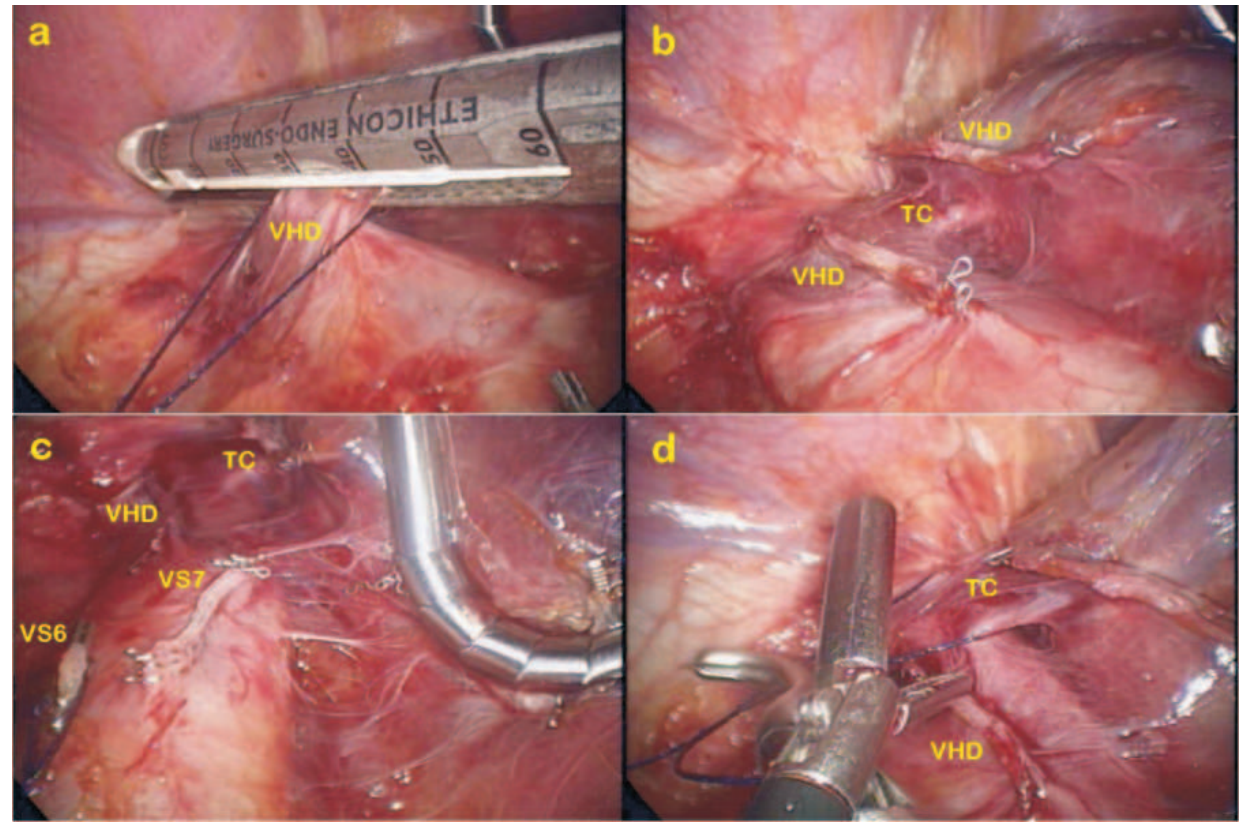

Figura 3 - Controle das veias hepáticas. a. Secção da veia hepática direita (VHD) com grampeador vascular. $b$. Aspecto após secção da VHD. O tronco comum (TC), formado pela união das veias hepáticas esquerda e média é vista ao fundo. c. Face anterior da veia cava retrohepática completamente exposta após seç̧ão da VHD e das veias acessórias dos segmentos 6 e 7 (VH6 E VH7). d. Tronco comum é circundado com fio de reparo para controle do retorno venoso. este procedimento pode ser realizado no Brasil, de maneira segura.

A fase mais difícil deste procedimento é a secção do parênquima que apresenta risco potencial de sangramento. $\mathrm{O}$ risco de sangramento é diminuído com o uso de pressão venosa central baixa e com o estabelecimento do pneumoperitônio em $12 \mathrm{mmHg}$.
A trisegmentectomia é um procedimento que envolve a remoção de $75 \%$ a $80 \%$ do volume hepático total, o que pode levar a insuficiência hepática pós-operatória. A embolização pré-operatória do ramo direito da veia porta tem como objetivo provocar uma hipertrofia compensatória do fígado remanescente (segmentos 2 e 3 ) e causar uma atrofia do lobo direito ${ }^{9}$. Com isto a trisegmentectomia pode ser realizada
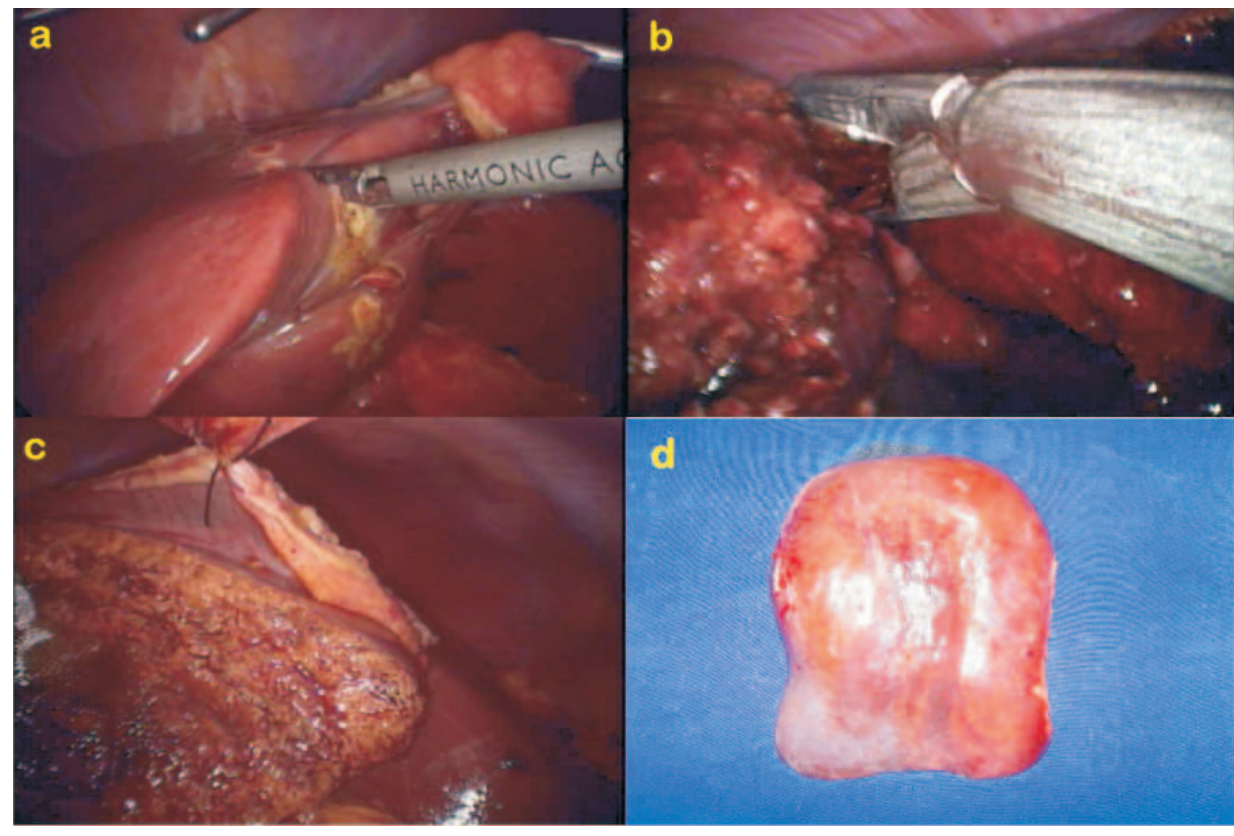

Figura 4 - Trisegmentectomia hepática direita por laparoscopia. a. Secção do parênquima inicia-se no segmento 4, 1 cm à direita do ligamento falciforme com bisturi harmônico. b.O fígado é seccionado com uma combinação de bisturi harmônico e grampeador endoscópico. c. Aspecto final após trisegmentectomia direita. Observe área cruenta próxima ao ligamento falciforme que foi suturado à parede abdominal. Segmentos 2 e 3 com coloração normal. d. Espécime cirúrgico. 
de maneira mais segura, assegurando ao paciente um volume hepático remanescente adequado.

Uma etapa fundamental para o sucesso desta intervenção é o correto posicionamento do paciente e a mobilização do fígado ${ }^{1}$. A colocação de coxim no flanco direito e o decúbito lateral esquerdo possibilitam que o fígado caia em direção ao hipocôndrio esquerdo e facilite a exposição da veia cava após a secção dos ligamentos triangular e lateral direito ${ }^{1}$. No entanto, após a liberação da veia cava retro-hepática, o paciente dever ser recolocado em posição supina, pois a secção do fígado será realizada junto ao ligamento falciforme que se localizada exatamente sobre a linha mediana do abdome. Para isso, utilizamos um trocarte de $5 \mathrm{~mm}$ na linha hemiclavicular esquerda e a ótica é introduzida no trocarte de $12 \mathrm{~mm}$ posicionado sobre a linha mediana.

Outro passo muito importante foi o controle do retorno venoso. A secção de todas as veias hepáticas acessórias juntamente com a veia hepática direita fez com que o fígado tivesse apenas o tronco comum (veia hepática média e esquerda) como veia de drenagem. A dissecção do tronco comum e seu reparo, durante a fase de secção do fígado, permitiram controle total do retorno venoso, diminuindo o risco de sangramento.

A técnica de acesso intra-hepático ao pedículo Glissoniano permite o controle rápido da tríade portal evitando a dissecção do hilo hepático ${ }^{10,11}$. O uso deste tipo de acesso é factível na ressecção hepática por laparoscopia ${ }^{5}$ e se mostrou muito útil na realização da trisegmentectomia direita.

Trisegmentectomia hepática direita por videolaparoscopia é procedimento factível e seguro e deve ser considerado para pacientes selecionados. Este procedimento deve ser realizado em centros especializados com experiência em cirurgia hepática e cirurgia laparoscópica avançada.

\begin{abstract}
Background: Laparoscopic right liver trisectionectomy is a very complex procedure and, to our knowledge, there is only one technical description so far in the English literature. The authors describe the first totally laparoscopic right trisectionectomy performed in Brazil. Method: Patient is placed in left semi-lateral decubitus position with surgeon standing between patients' legs. Five trocars, three $12 \mathrm{~mm}$ and two $5 \mathrm{~mm}$, were used. Due to previous right portal vein embolization, hepatic pedicle is not dissected. Intrahepatic access to the main right Glissonian pedicle is achieved with two small incisions: on the right portion of caudate lobe and another in front of the hilum. A vascular stapling device is inserted between these incisions and fired. Right liver is then mobilized and inferior vena cava is dissected. Right hepatic vein is divided with vascular endoscopic stapler. Line of liver transection is marked along the liver surface including segment 4. Glissonian pedicle from segment 4 is divided during liver transection. Liver transection is accomplished with harmonic scalpel and endoscopic stapling device as appropriate. Specimen is extracted through a suprapubic incision and pneumoperitoneum is reestablished. Raw surface area is checked for hemostasia and biliary leakage. One round abdominal drain is left in place. Right hepatic trisectionectomy is then completed. Conclusion: Totally laparoscopic right trisectionectomy is safe and feasible in selected patients and should be considered for patients with benign or malignant liver neoplasms. However, this complex procedure should be performed by surgeons who have both experience in advanced laparoscopic procedures and open hepatic surgery.
\end{abstract}

Key words: Liver; Laparoscopy; Right Trisectionectomy; Anatomy; Technique.

\section{REFERÊNCIAS}

1. Machado MA, Makdissi FF, Surjan RC, Teixeira AR, Bacchella T, Machado MC. Hepatectomia direita por videolaparoscopia. Rev Col Bras Cir. 2007; 34(3):189-92.

2. Soubrane O, Cherqui D, Scatton O, Stenard F, Bernard D, Branchereau S, Martelli H, Gauthier F. Laparoscopic left lateral sectionectomy in living donors: safety and reproducibility of the technique in a single center. Ann Surg. 2006; 244(5):815-20.

3. O'Rourke N, Fielding G. Laparoscopic right hepatectomy: surgical technique. J Gastrointest Surg. 2004; 8(2):213-6.

4. Koffron AJ, Auffenberg G, Kung R, Abecassis M. Evaluation of 300 minimally invasive liver resections at a single institution: less is more. Ann Surg. 2007; 246(3):385-92; discussion 392-4.

5. Machado MA, Makdissi FF, Galvão FH, Machado MC. Intrahepatic Glissonian approach for laparoscopic right segmental liver resections. Am J Surg. 2008; 196(4):e38-42. Epub 2008 Jul 9.

6. Halazun KJ, Al-Mukhtar A, Aldouri A, Malik HZ, Attia MS, Prasad KR, Toogood GJ, Lodge JP. Right hepatic trisectionectomy for hepatobiliary diseases: results and an appraisal of its current role. Ann Surg. 2007; 246(6):1065-74.

7. Gumbs AA, Gayet B. Totally laparoscopic extended right hepatectomy. Surg Endosc. 2008; 22(9):2076-7. Epub 2008 Jun 14.
8. Ogata S, Kianmanesh R, Belghiti J. Doppler assessment after right hepatectomy confirms the need to fix the remnant left liver in the anatomical position. Br J Surg. 2005; 92(5):592-5.

9. Hemming AW, Reed AI, Howard RJ, Fujita S, Hochwald SN, Caridi JG, Hawkins IF, Vauthey JN. Preoperative portal vein embolization for extended hepatectomy. Ann Surg. 2003; 237(5):686-91; discussion 691-3.

10. Martins AC, Machado MA. O acesso glissoniano intra-hepático nas ressecções do fígado. Rev Col Bras Cir. 2008; 35(1):45-50.

11. Machado MA, Herman P, Machado MC. Standardized technique for right segmental liver resections. Arch Surg. 2003;138(8):918-20.

Como citar este artigo:

Machado MA, Makdissi FF, Surjan RC, Oliveira AC, Pilla VF. Trisegmentectomia hepática direita por videolaparoscopia. Rev Col Bras Cir. [periódico na Internet] 2008; 35(5). Disponível em URL: http://www.scielo.br/rcbc

Endereço para correspondência:

Marcel Autran C. Machado

Rua Dona Adma Jafet 74 cj 102

01308-050 - São Paulo - SP

Tel: (11) 3256-4098 Fax: (11) 3259-8231

www.drmarcel.com.br / E-mail:dr@drmarcel.com.br 